\title{
Physical Examination Findings of New or Worsening Heart Failure
}

National Cancer Institute

\section{Source}

National Cancer Institute. Physical Examination Findings of New or Worsening Heart

Failure. NCI Thesaurus. Code C119233.

A description of the physical examination evidence of heart failure that can be classified as new or worsening. 